EESTI NSV TEADUSTE AKADEEMIA TOIMETISED. 25. KOIDE FOUSIKA * MATEMAATIKA. 1976, NR. 2

ИЗВЕСТИЯ АКАДЕМИИ НАУК ЭСТОНСКОИ ССР. ТОМ 25 ФИЗНҚА * МАТЕМАТИҚА, 1976, № 2

\title{
ОБ ОДНОМ ПРИМЕРЕ Э. ДУБИНСКОГО И ДЖ. РЕЗЕРФОРДА
}

Если шаудерово разложение $\left(P_{n}\right)$ локально выпуклого пространства является полным и простым, то справедлива импликация: $(D)$ из натягиваемости сопряженного разложения $\left(P_{n}{ }^{\prime}\right)$ следует ограниченная полнота $\left(P_{n}\right)$ [ $\left.{ }^{1}\right]$. В настоящем сообщении опровергается утверждение авторов $\left[{ }^{2}\right]$ о том, что построенный ими пример представляет собой контрпример к $(D)$. Приводится простой пример, показываюшций, что $(D)$ действительно не переносится на произвольные шаудеровы разложения.

1. Ш а удеровы м р аз ло жен и е м (отделимого) локально выпуклого пространства $X$ называется последовательность * $\left(P_{n}\right)$ непрерывных ненулевых проекторов в пространстве $X$ такая, что $P_{n} \circ P_{m}=0$, если $n \neq m$, и любой элемент $x \in X$ представим в виде $x=\Sigma P_{n} x$, где ряд сходится в топологии пространства $X$. Шаудерово разложение $\left(P_{n}\right)$ пространства $X$ называется ограниченно полным (соответственно полным), если каждая ограниченная последовательность (соответственно последовательность Коши) $\left(\Sigma_{1 \leqslant k \leqslant n} x_{k}\right), \quad$ где $x_{k} \in P_{k} X$, сходится. Если последовательность сопряженных отображений $\left(P_{n}{ }^{\prime}\right)$ образует шаудерово разложение для сильного сопряженного $\left(X^{\prime}, \beta\left(X^{\prime}, X\right)\right)$ к $X$, то шаудерово разложение $\left(P_{n}\right)$ пространства $X$ называется натягив а щи м. Шаудерово разложение $\left(P_{n}\right)$ пространства $X$ называется простым (соответственно безусловно п ростым), если для любого функционала $f \in X^{\prime}$ множество $\left\{\Sigma_{1 \leqslant k \leqslant n} P_{k}^{\prime} f: n=1,2, \ldots\right\}$ (соответственно $\left\{\Sigma_{k \in v} P_{k}^{\prime} f: v \in \Sigma\right\}$ где $\Sigma$ семейство всех конечных множеств натуральных чисел) сильно (т. е. $\beta\left(X^{\prime}, X\right)$-) ограничено.

Шаудеров базис $\left(e_{n}\right) \subset X$ с сопряженной последовательностью $\left(f_{n}\right) \subset X^{\prime}$ представляет собой шаудерово разложение пространства $X$ на одномерные подпространства $P_{n} X$, где проекторы $P_{n}$ определены равенствами $P_{n} x=f_{n}(x) e_{n}, x \in X$.

2. Если $\left(P_{n}\right)$ - шаудерово разложение локально выпуклого пространства $X$, то, очевидно, последовательность $\left(P_{n}^{\prime}\right)$ (точнее, последовательность сужений проекторов $P_{n}{ }^{\prime}$ ) является шаудеровым разложением для пространства $H=\left\{f \in X^{\prime}: f=\Sigma P_{n}^{\prime} f\right.$ в топологии $\left.\beta\left(X^{\prime}, X\right)\right\}$, наделенного топологией, индуцированной $\beta\left(X^{\prime}, X\right)$. В $\left.{ }^{1}\right]$ было доказано, что для полного простого шаудерова разложения $\left(P_{n}\right)$ в локально выпуклом пространстве $X$ (в частности, для любого шаудерова разложе-

* Если пределы изменения индексов не указаны, значит, они пробегают все значения $1,2, \ldots$. 
ния в секвенциально полном локально выпуклом пространстве) справедлива импликация: $(D)$ если шаудерово разложение $\left(P_{n}^{\prime}\right)$ для $H$ натягивающее, то $\left(P_{n}\right)$ ограниченно полно.

В этом сообщении докажем ошибочность утверждения Э. Дубинского и Дж. Резерфорда $\left.{ }^{2}\right]$ (c. 272 ) о том, что для шаудерова базиса ** $e_{k}=\left(\delta_{k n}\right)$ в пространстве *** $X=(\Phi, \sigma(\Phi, \Phi))$, где $\Phi$ - линейное множество всех финитных числовых последовательностей, импликация $(D)$ не справедлива. Кроме того, приведем пример такого шаудерова базиса, для которого $(D)$ не верна.

3. Рассмотрим вышеприведенный пример Э. Дубинского и Дж. Резерфорда. Базис $\left(e_{k}\right)$, очевидно, не ограниченно полный, так как, например, последовательность $\left(\Sigma_{1 \leqslant k \leqslant n} e_{k}\right)$ ограничена, но не сходится. Ясно, что $H=X^{\prime}=\Phi$. Авторы [2] утверждают, что сопряженный базис $f_{k}=\left(\delta_{k n}\right)$ для $H$ натягивающий. Покажем, что их утверждение ошибочно.

Поскольку при всех $f \in X^{\prime}$ множество $\left\{\Sigma_{k \in v} P_{k}^{\prime} f: v \in \Sigma\right\}$ конечно и, значит, сильно ограничено, стало быть, базис $\left(e_{k}\right)$ безусловно простой.

Для исследования сопряженного базиса необходимо знать описание ограниченных подмножеств в $X$. Так как $\left(e_{k}\right)$ безусловно простой, в силу теоремы 1.5 из $\left[{ }^{3}\right]$ семейство всех ограниченных подмножеств в

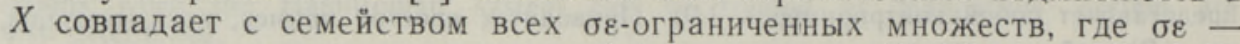
$\varepsilon$-топология, соответствующая слабой топологии $\sigma\left(X, X^{\prime}\right)\left(\right.$ см. $\left.\left[{ }^{3}\right], \S 1\right)$. Поэтому для любого ограниченного в $X$ множества $A$ с помощью неравенства (4) из $\left[{ }^{3}\right]$ при всех $k$ получаем

$$
\sup _{x=\left(x_{n}\right) \in A} \sup _{n}\left|x_{n}\right| \cdot \sup _{x=\left(x_{n}\right) \in A} \sup _{n}\left|f_{k}\left(\delta\left(x_{n}\right) e_{n}\right)\right| \leqslant \sup _{x \in A} q_{f_{k}}(x)<\infty,
$$

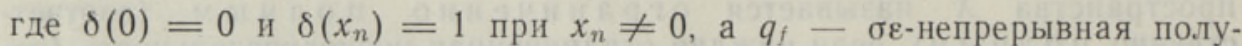
норма, соответствующая согласно формуле (2) из $\left[{ }^{3}\right]$ полунорме $|f(\cdot)|$, $f \in X^{\prime}$. Отсюда видно, что

$$
\sup _{x=\left(x_{n}\right) \in A} \sup _{n}\left|x_{n}\right|<\infty,
$$

если множество $A$ ограничено в $X$. Обратно, если выполнено условие (1), то в силу неравенства (3) из $\left[{ }^{3}\right]$ и того, что последовательность

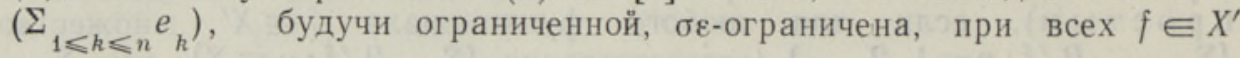
имеем

$$
\sup _{x \in A}|f(x)| \leqslant 2 \sup _{x=\left(x_{n}\right) \in A} \sup _{n}\left|x_{n}\right| \cdot \sup _{n} q_{f}\left(\sum_{k=1}^{n} e_{k}\right)<\infty,
$$

т. е. множество $A$ ограничено в $X$. Таким образом, подмножество $A$ в $X$ ограничено тогда и только тогда, когда оно удовлетворяет условию (1).

Рассмотрим $e=(1,1, \ldots)$. Поскольку при всех $h=\left(h_{k}\right) \in H$

$$
|e(h)|=\left|\Sigma h_{k}\right| \leqslant \Sigma\left|h_{h}\right|=\left(\Sigma h_{k} f_{k}\right)\left(\Sigma s_{k} e_{k}\right) \leqslant \sup _{x \in E}|h(x)|,
$$

где $s_{k}=\operatorname{sgn} h_{k}$ и $E=\left\{\Sigma_{k \in v} \varepsilon_{k} e_{k}: \varepsilon_{k}= \pm 1, v \in \Sigma\right\}$, а множество $E$ ограничено в $X$, то $e \in H^{\prime}$. Ясно, что $\left(e_{k}\right) \subset H^{\prime}$ и последовательность

** Пусть $\delta_{k k}=1$ и $\delta_{k n}=0$ при $\dot{k} \neq n$.

*** Двойственность (Ф, $\Psi)$ между линейными множествами числовых последовательностей $\Phi$ и $\Psi$ определяется билинейной формой $(\varphi, \psi) \rightarrow \Sigma \varphi_{n} \psi_{n}, \quad$ где $\varphi=$ $=\left(\varphi_{n}\right) \in \Phi$ и $\psi=\left(\psi_{n}\right) \in \Psi$. 
$\left(\Sigma_{1 \leqslant k \leqslant n} e_{k}\right)$ сходится к $е$ в $\sigma\left(H^{\prime}, H\right)$. Но так как при всех $n$

$$
\sup _{m}\left|\left(e-\sum_{k=1}^{n} e_{k}\right)\left(f_{m}\right)\right|=1,
$$

а множество $\left\{f_{m}: m=1,2, \ldots\right\} \subset X^{\prime}$ является сильно ограниченным (поскольку

$$
\sup _{m} \sup _{x \in A}\left|f_{m}(x)\right|=\sup _{x=\left(x_{n}\right) \in A} \sup _{n}\left|x_{n}\right|<\infty
$$

для любого ограниченного множества $A$ из $X)$, то $\left(\Sigma_{1 \leqslant k \leqslant n} e_{k}\right)$ не сходится к $е$ в $\beta\left(H^{\prime}, H\right)$. Следовательно, базис $\left(f_{k}\right)$ пространства $H$ не является натягивающим и тем самым для базиса $\left(e_{k}\right)$ пространства $X$ импликация $(D)$ все же справедлива.

Э. Дубинский и Дж. Резерфорд допустили ошибку, полагая, что $H=\left(X^{\prime}, \beta\left(X^{\prime}, X\right)\right)$ - подпространство в пространстве Фреше $\omega$ всех числовых последовательностей. (В действительности топология, которую $\omega$ индуцирует на $X^{\prime}$, строго слабее****, чем $\beta\left(X^{\prime}, X\right)$ : последовательность $\left(\Sigma_{1 \leqslant k \leqslant n} f_{k}\right)$ сходится в $\omega$, но в $\beta\left(X^{\prime}, X\right)$ она даже не ограничена, так как она не ограничена, например, на множестве $\left\{\Sigma_{1 \leqslant k \leqslant n} e_{k}: n=1,2, \ldots\right\}$.) Поскольку $H=\Phi$ всюду плотно в $\omega$, авторы $\left[{ }^{2}\right]$ пришли к выводу, что $H^{\prime}=\Phi$. т. е. $\left(f_{h}\right)$ для $H$ натягиваюший. Следует отметить, что равенство $H^{\prime}=\Phi$ приводит к противоречию, ибо из него вытекает полурефлексивность пространства $X$. А шаудеров базис полурефлексивного пространства всегда ограниченно полный (см., напр., $\left.\left[{ }^{4}\right]\right)$. Однако, как мы видели, базис $\left(e_{k}\right)$ для $X$ не ограниченно полный.

4. Приведем пример безусловно простого шаудерова базиса, для которого утверждение $(D)$ не верно. Пусть $X$ - линейное множество всех финитных числовых последовательностей, наделенное $l_{1}$-нормой. Последовательность $e_{k}=\left(\delta_{k n}\right)$ является для $X$ безусловным шаудеровым базисом. Поскольку, очевидно, $\left\|\Sigma_{k \in v} P_{h}^{\prime}\right\|=\left\|\Sigma_{k \in v} P_{k}\right\|=1$ для всех $v \in \Sigma$, то базис $\left(e_{k}\right)$ безусловно простой.

Нетрудно проверить, что $H=c_{0}$. Следовательно, сопряженный базис $f_{k}=\left(\delta_{k n}\right)$ для $H$ натягивающий. С другой стороны, ясно, что базис $\left(e_{k}\right)$ не полный, а значит, и не ограниченно полный.

\footnotetext{
**** То, что топология, которую $\omega$ индуцирует на $X^{\prime}$, слабее, чем $\beta\left(X^{\prime}, X\right)$, ясно из сильной непрерывности отображения, сопряженного к тождественному отображению $I: \quad(\Phi, \sigma(\Phi, \omega)) \rightarrow(\Phi, \sigma(\Phi, \Phi))$.
}

\section{ЛИ ТЕРАТУРА}

1. О я Э., Уч. зап. Тартуск. гос, ун-та, 374, 117 (1975).

2. Dubinsky E., Ret herford J. R., Trans. Amer. Math. Soc., 130, No. 2, 265 (1968).

3. О я Э., Уч. зап. Тартуск. гос. ун-та, 374,90 (1975).

4. Cook T. A., Math. Ann., 182, No. 3, 232 (1969).

Тартуский государственный университет
Поступила в редакщию 3/VII 1975

Eve OJA

\section{OHEST E. DUBINSKY JA J. R. RETHERFORDI NÄITEST}

Olgu $\left(P_{n}\right)$ eralduva lokaalselt kumera ruumi $X$ Schauderi lahutus. Kui $H=$ $=\left\{f \in X^{\prime}: f=\Sigma P_{n}^{\prime} f\right.$ topoloogias $\left.\beta\left(X^{\prime}, X\right)\right\}$ varustada topoloogia $\beta\left(X^{\prime}, X\right)$ poolt indutseeritud topoloogiaga, siis $\left(P_{n}^{\prime}\right)$ on ruumi $H$ Schauderi lahutus. Vaatleme järgmist väidet: $(D)$ kui $\left(P_{n}^{\prime}\right)$ on $H$ jaoks pingul, siis $\left(P_{n}\right)$ on tôkestatult täielik. 
Implikatsioon $(D)$ on tõene, kui Schauderi lahutus $\left(P_{n}\right)$ on täielik ja lihtne [1]. Artiklis näidatakse, et $\left[{ }^{2}\right], 1 \mathrm{k} .272$, toodud näite korral on $(D)$ tõene. Järelikult ei kujuta see näide endast kontranäidet väite $(D)$ üldisele kehtivusele. Tuuakse ka näide, mille korral $(D)$ tõepoolest ei kehti.

\section{Eve OJA}

\section{ON AN EXAMPLE OF E. DUBINSKY AND J. R. RETHERFORD}

Let $\left(P_{n}\right)$ be a Schauder decomposition for a locally convex (Hausdorff) space $X$. Then $\left(P_{n}^{\prime}\right)$ is a Schauder decomposition for $H=\left\{f \in X^{\prime}: f=\Sigma P_{n}{ }^{\prime}\right.$ where the series converges in the strong topology $\left.\beta\left(X^{\prime}, X\right)\right\}$ where $H$ is equipped with the topology induced by $\beta\left(X^{\prime}, X\right)$. We consider the following statement: $(D)$ if $\left(P_{n}^{\prime}\right)$ is shrinking for $H$ then $\left(P_{n}\right)$ is boundedly complete.

In $\left[{ }^{1}\right]$ we proved that for a complete simple Schauder decomposition $\left(P_{n}\right)$ in a locally convex space $X$ the implication $(D)$ is true. In this paper we show that for the example given in $\left[{ }^{2}\right]$, p. $272,(D)$ is true, and so the example actually is not a counterexample for the implication $(D)$. We also give a counterexample for $(D)$. Hence, in general, the implication $(D)$ fails to hold. 